\title{
Unification of Treatments and Interventions for Tinnitus Patients (UNITI): a study protocol for a multi-center randomized clinical trial
}

Stefan Schoisswohl ${ }^{1 *}$ D, Berthold Langguth1, Martin Schecklmann', Alberto Bernal-Robledano², Benjamin Boecking ${ }^{3}$, Christopher R. Cederroth ${ }^{4}$, Dimitra Chalanouli ${ }^{5}$, Rilana Cima ${ }^{6}$, Sam Denys ${ }^{7,8,9}$, Juliane Dettling-Papargyris ${ }^{10}$, Alba Escalera-Balsera ${ }^{11}$, Juan Manuel Espinosa-Sanchez ${ }^{2,11}$, Alvaro Gallego-Martinez ${ }^{11}$, Efi Giannopoulou ${ }^{5}$, Leyre Hidalgo-Lopez ${ }^{12}$, Michael Hummel ${ }^{13}$, Dimitris Kikidis ${ }^{14}$, Michael Koller ${ }^{15}$, Jose A. Lopez-Escamez ${ }^{2,11,16}$, Steven C. Marcrum ${ }^{17}$, Nikolaos Markatos ${ }^{14}$, Juan Martin-Lagos ${ }^{18}$, Maria Martinez-Martinez ${ }^{2,11}$, Marta Martinez-Martinez ${ }^{2,18}$, Maria Mata Ferron ${ }^{2,11}$, Birgit Mazurek ${ }^{3}$, Nicolas Mueller-Locatellii ${ }^{18}$, Patrick Neff ${ }^{1,19}$, Kevin Oppel ${ }^{10}$, Patricia Perez-Carpena ${ }^{2,11}$, Paula Robles-Bolivar ${ }^{11}$, Matthias Rose ${ }^{20}$, Tabea Schiele ${ }^{3}$, Axel Schiller ${ }^{1}$, Jorge Simoes ${ }^{1}$, Sabine Stark ${ }^{3}$, Susanne Staudinger ${ }^{1}$, Alexandra Stege $^{13}$, Nicolas Verhaert ${ }^{7,8,9}$ and Winfried Schlee ${ }^{1}$

\begin{abstract}
Background: Tinnitus represents a relatively common condition in the global population accompanied by various comorbidities and severe burden in many cases. Nevertheless, there is currently no general treatment or cure, presumable due to the heterogeneity of tinnitus with its wide variety of etiologies and tinnitus phenotypes. Hence, most treatment studies merely demonstrated improvement in a subgroup of tinnitus patients. The majority of studies are characterized by small sample sizes, unstandardized treatments and assessments, or applications of interventions targeting only a single organ level. Combinatory treatment approaches, potentially targeting multiple systems as well as treatment personalization, might provide remedy and enhance treatment responses. The aim of the present study is to systematically examine established tinnitus therapies both alone and in combination in a large sample of tinnitus patients. Further, it wants to provide the basis for personalized treatment approaches by evaluating a specific decision support system developed as part of an EU-funded collaborative project (Unification of treatments and interventions for tinnitus patients; UNITI project).
\end{abstract}

\footnotetext{
* Correspondence: stefanschoisswohl@yahoo.de

'Department of Psychiatry and Psychotherapy, University of Regensburg, Universitaetsstraße 84, 93053 Regensburg, Germany

Full list of author information is available at the end of the article
}

(c) The Author(s). 2021 Open Access This article is licensed under a Creative Commons Attribution 4.0 International License, which permits use, sharing, adaptation, distribution and reproduction in any medium or format, as long as you give appropriate credit to the original author(s) and the source, provide a link to the Creative Commons licence, and indicate if changes were made. The images or other third party material in this article are included in the article's Creative Commons licence, unless indicated otherwise in a credit line to the material. If material is not included in the article's Creative Commons licence and your intended use is not permitted by statutory regulation or exceeds the permitted use, you will need to obtain permission directly from the copyright holder. To view a copy of this licence, visit http://creativecommons.org/licenses/by/4.0/ The Creative Commons Public Domain Dedication waiver (http://creativecommons.org/publicdomain/zero/1.0/) applies to the data made available in this article, unless otherwise stated in a credit line to the data. 
Methods/study design: This is a multi-center parallel-arm randomized clinical trial conducted at five different clinical sites over the EU. The effect of four different tinnitus therapy approaches (sound therapy, structured counseling, hearing aids, cognitive behavioral therapy) applied over a time period of 12 weeks as a single or rather a combinatory treatment in a total number of 500 chronic tinnitus patients will be investigated. Assessments and interventions are harmonized over the involved clinical sites. The primary outcome measure focuses on the domain tinnitus distress assessed via the Tinnitus Handicap Inventory.

Discussion: Results and conclusions from the current study might not only provide an essential contribution to combinatory and personalized treatment approaches in tinnitus but could also provide more profound insights in the heterogeneity of tinnitus, representing an important step towards a cure for tinnitus.

Trial registration: ClinicalTrials.gov NCT04663828. Registered on 11 December 2020.

Keywords: Tinnitus, Treatment, Hearing aids, Cognitive behavioral therapy, Sound therapy, Structured counseling, Multi-center, RCT

\section{Background}

Following a recent multidisciplinary consensus, tinnitus is termed as "the conscious awareness of a tonal or composite noise for which there is no identifiable corresponding external acoustic source" [1] whereby a continuous perception over 6 months constitutes a chronification [2]. Frequent causes for the emergence of tinnitus range from noise trauma, presbyacusis to intake of ototoxic medication, potentially provoking pathological neural alterations in the central auditory pathway [3]. Approximately $10-15 \%$ of the global adult population are affected by this phantom sound perception [4], whereas $2-3 \%$ are particularly suffering from tinnitus $[5,6]$. In many cases, tinnitus generates a high level of suffering and can be accompanied by various comorbidities such as depression, anxiety, or sleep disorders [7-10], explicitly defined as tinnitus disorder [1]. Currently, there is no general treatment respectively a cure for tinnitus existent. Available treatment approaches cover a broad spectrum of interventions from pharmacology [6], neurostimulation [11], cochlear implants [12] to different sound therapies $[13,14]$ or hearing aids [15, 16]. While the European guidelines for tinnitus [17] give a weak recommendation for the application of amplification devices in tinnitus patients with hearing loss, there is currently no explicit recommendation for pharmacological interventions or neurostimulation [17]. Up to now, cognitive behavioral therapy approaches exhibit the best body of evidence for the treatment of tinnitus [18, 19] with a strong recommendation according to European guidelines [17]. Even though tinnitus research has been impressively expanded over the past decade, the majority of studies suffers from methodological shortcomings such as heterogeneous patient samples, imprecisely defined therapeutic interventions, relatively small sample sizes, and a lack of predefined primary outcomes and data analysis strategies [20, 21]. Beyond tackling these limitations in prospective studies, interdisciplinary multi-center randomized clinical trials (RCT) could help to further increase the validity and interpretability of results.

The complexity of tinnitus with a wide variety of phenotypes and different etiologies plus the uncertainty about underlying pathophysiological processes make the quest in finding an appropriate treatment rather difficult $[3,22]$. In most of the studies, only a subgroup of patients exhibits improvement to a certain intervention ranging from $1 \%$ to more than $35 \%$. However, currently none of the treatment approaches has sound and universal findings [23]. Thus a common treatment for all tinnitus subtypes is most unlikely, highlighting the necessity of so-called precision medicine or rather personalized treatment concepts in tinnitus $[24,25]$. A potential proceeding would be the identification of demographic or tinnitus-related characteristics, potentially capable to predict a patient's response to a certain type of intervention [26]. Such predictive markers could facilitate a so-called Decision Support System (DSS), which could assist clinicians in selecting the most promising tinnitus treatment on an individual patient level [20].

Currently available tinnitus treatments mostly aim at different systems and domains, e.g., the auditory system (AS) or the central nervous system (CNS) respectively focuses mainly on a single target of the underlying pathophysiology. At best, tinnitus treatments should integrate all involved components/ systems [27]; hence, a combination of different therapeutic approaches could provide further remedy. Studies focusing on combinatory interventions range from combinations of hearing aids with sound generators [28-30], simultaneous sound and somatosensory stimulations [31,32], application of counseling together with tinnitus masking termed tinnitus retraining therapy $[13,22,33,34]$, to brain stimulation in combination with relaxation [35] as well as 
multimodal therapies [36, 37], though past studies are not capable to provide a clear superiority of combined interventions. A systematic examination of several different single and combinational interventions is currently not available, highlighting the need for more profound investigations in this regard.

The present study protocol describes the methodological procedure of the UNITI-RCT (Unification of Treatments and Interventions for Tinnitus Patients Randomized Clinical Trial), which constitutes the centerpiece of the EU-funded UNITI project. The primary objective of the UNITI project is the development of a computational model to predict patients' responses to distinct treatments in order to facilitate personalized therapies in tinnitus. For a detailed overview of goals and procedures of the UNITI project, see Schlee et al. [38].

\section{Objectives}

The attempt of the UNITI-RCT is to not only overcome the shortcomings of previous studies, but also pave the way for personalized medicine approaches in tinnitus. For this purpose, a multi-center parallel-arm superiority RCT, implemented and harmonized among five clinical sites across the EU, combining and investigating selected existing therapies evaluated in the European guidelines for tinnitus [17], is conducted. The main objective of the UNITI-RCT is (1) to scrutinize if a combinational therapy is more effective than a single therapy for the treatment of chronic tinnitus. Additionally, (2) the outcomes of each utilized intervention will be compared against each other; (3) several treatment groups will be formed and analyzed based on whether participants received a certain treatment type alone or in combination with another treatment, (4) whether participants received a certain type of intervention at all (either alone or in combination), and (5) whether the received interventions targeted on one or two organ levels - the ear or the CNS (ear- vs. brain-mediated interventions). Moreover, (6) the development of a specific DSS, which will be based on demographic, psychological, audiological, electrophysiological, and genetic parameters, for patientspecific data-driven treatment suggestions [39] will be validated over the course of the UNITI-RCT.

\section{Methods}

For the reporting of the methodological approach of the UNITI-RCT, the SPIRIT guidelines have been used [40].

\section{Study design}

The study is designed as a multi-center RCT which investigates the effect of four different tinnitus therapy approaches applied as single or combinatory treatments over a time period of 12 weeks in 500 patients with mild to severe tinnitus distress. The clinical trial has been registered at ClinicalTrials.gov (NCT04663828; trial registration dataset in the supplemental material) and will be completed in five different clinical sites across the European Union.

(1) University of Regensburg, Regensburg, Germany (RCT coordinator)

(2) Charité - Universitaetsmedizin Berlin, Berlin, Germany

(3) Ethniko Kai Kapodistriako Panepistimo Athinon, Athens, Greece

(4) Hospital Universitario Virgen de las Nieves/Hospital Clinico Universitario San Cecilio, Granada, Spain

(5) Katholieke Universiteit Leuven, Leuven, Belgium

\section{Sample size determination/ effect size calculation}

Since there is no reliable data for the main purpose of the study (single vs. combinational treatment) available from which an effect size for sample calculation can be deduced, literature from a stepped-care approach involving counseling, sound therapy, and CBT was used for this purpose [18]. This study exhibited an effect size of 0.52 after 8 months when a stepped-care approach including a combination of treatments was contrasted against treatment as usual. This approach is not fully comparable to the current RCT (single vs. combinational treatment), as also treatments with much lower effect sizes are included in the current study (hearing aids, sound therapy, structured counseling), and effect size was estimated conservatively as about 0.26 . With a significance level of $5 \%$ and a power of $80 \%$ (two-sided test), the necessary sample size is 468 . Taking also dropouts into account, the aim is to investigate a total sample size of $N=500$.

\section{Study population}

As already mentioned above a total number of 500 participants will be investigated in the course of this RCT. Each of the five clinical sites has the objective to examine $n=100$ patients with chronic subjective tinnitus for the RCT with respect to specific inclusion and exclusion criteria. Potential candidates will be recruited via media advertising (according to local regulations) as well as on an individual basis at the clinical sites through, e.g., information sheets, word of mouth, or in conversations with medical staff. In order to be eligible for participation, potential participants have to meet the following criteria as outlined in Table 1.

\section{Outcome measures and assessments}

All measures, assessments, and documentations are harmonized among the clinical sites. Besides the below listed standardized measures and assessments, 
Table.1 Eligibility criteria

\begin{tabular}{|c|c|}
\hline Inclusion criteria & Exclusion criteria \\
\hline $\begin{array}{l}\text { - Primary complaint tinnitus } \\
\text { - Chronic tinnitus ( } \geq 6 \text { months) } \\
\text { - Age between } 18 \text { and } 80 \text { years } \\
\text { - A score of } \geq 18 \text { in the Tinnitus Handicap Inventory (THI; [41] - at least } \\
\text { mild tinnitus distress } \\
\text { - A score of }>22 \text { in the Montreal Cognitive Assessment (MoCa; [52])- } \\
\text { absence of mild cognitive impairment } \\
\text { - Ability and willingness to use the UNITI mobile applications on } \\
\text { smartphones } \\
\text { - Openness to use a HA (if indication and allocation to HA group) } \\
\text { - Ability to understand and consent to the research (hearing ability, } \\
\text { intellectual capacity) } \\
\text { - Ability to participate in all relevant visits (no plans for, e.g., long-term } \\
\text { holidays or pregnancy } \\
\text { - Existing drug therapies with psychoactive substances (e.g., } \\
\text { antidepressants or anticonvulsants) must be stable for at least } 30 \text { days at } \\
\text { the beginning of the therapeutic intervention. The drug therapy should } \\
\text { remain constant during the course of the study. Necessary changes do } \\
\text { not constitute an exclusion criterion per se, but need to be recorded. }\end{array}$ & $\begin{array}{l}\text { - Objective tinnitus or heartbeat-synchronous tinnitus as primary } \\
\text { complaint } \\
\text { - Otosclerosis/ acoustic neuroma or other relevant ear disorders with } \\
\text { fluctuation hearing } \\
\text { - Present acute infections (acute otitis media, otitis externa, acute } \\
\text { sinusitis) } \\
\text { - Meniere's disease or similar syndromes (but not vestibular migraine) } \\
\text { - Serious internal, neurological or psychiatric conditions } \\
\text { - Epilepsy or other disorders of the central nervous system (e.g., brain } \\
\text { tumor or encephalitis) } \\
\text { - Clinically relevant drug, medication or alcohol abuse up to } 12 \text { weeks } \\
\text { before study start } \\
\text { - Severe hearing loss-inability to communicate properly in the course of } \\
\text { the study } \\
\text { - One deaf ear } \\
\text { - Missing written informed consent } \\
\text { - Start of any other tinnitus-related treatments, especially hearing aids, } \\
\text { structured counseling, sound therapy (with special devices; expecting } \\
\text { long-term effects) or cognitive behavioral therapy in the last } 3 \text { months } \\
\text { before the start of the study }{ }^{b}\end{array}$ \\
\hline
\end{tabular}

a Due to specific standards of the local ethics committee at the clinical site in Granada, Spain , with respect to the conduction of RCTs, all female participants will be tested with regard to an existing pregnancy

${ }^{b}$ If a HA has already been worn 3 months before screening, eligible candidates are allowed to participate, but are automatically assigned to the group with no HA indication

participants' comorbidities as well as concomitant treatments and medications will be recorded. All types of medication (even over-the-counter drugs), which have been ingested within the last 3 months before screening respectively up to the time of study starting, and which will be ingested during the study are documented with respect to dose, administration, and begin and stop date. Further, all types of treatments which have been performed within the last 3 months before screening respectively up to the time of study starting and/or will be performed during the course of the trial are documented with respect to frequency and begin/ stop date.

\section{Primary outcome}

The primary outcome of the current RCT is focused on the domain tinnitus distress. Changes in tinnitus distress with respect to the applied interventions will be assessed via the total score of the Tinnitus Handicap Inventory (THI) [41].

\section{Secondary outcome}

Several other standardized tinnitus- and health-related questionnaires will be used as secondary outcomes in the course of this RCT:

(1) Tinnitus Functional Index (TFI) [42]

(2) Mini Tinnitus Questionnaire (Mini-TQ) [43]

(3) Tinnitus numeric rating scales (NRS) [44]

(4) World Health Organization - Quality of Life abbreviated (WHOQoL-Bref; https://www.who.int/ healthinfo/survey/WHOQOL_BREF.pdf?ua=1)
(5) Clinical Global Impression Scale - Improvement (CGI-I) [45]

(6) Patient Health Questionnaire for Depression (PHQD/PHQ-9) [46, 47]

\section{Sample description and other measures}

(1) European School of Interdisciplinary Tinnitus Research Screening Questionnaire (ESIT-SQ) [48]

(2) Tinnitus Sample Case History Questionnaire (TSCHQ) [49]

(3) Questionnaire on Hypersensitivity to Sound (GUF) [50]

(4) Big Five Inventory-2 (BFI-2) [51]

(5) Montreal Cognitive Assessment (MoCA) [52]

(6) Social Isolation Electronic Survey (SOISES) [53]-a subset of 11 questions sensitive for tinnitus distress change will be used, further designated as MiniSOISES

(7) Attitudes Towards Amplification Questionnaire (ATAQ) - a subset of questions (baseline, 8; end of treatment, 7) associated with hearing aids taken from the Attitudes towards Loss of Hearing Questionnaire (ALHQ) [54]

(8) Fear of Tinnitus Questionnaire (FTQ) [55]

\section{Electrophysiological measures}

Two types of electrophysiological measures are performed in the course of this RCT-auditory brain stem responses $(\mathrm{ABR})$ and auditory middle latency responses (AMLR). ABRs represent the synchronized neural activity along the auditory pathway evoked by a serial 
presentation of acoustic stimuli (e.g., clicks). ABR is considered to be a robust electrophysiological method to assess the functional integrity of the auditory pathways. Normally, up to five waves occur in the ABR response. The wave I of the ABR reflects activity of the spiral ganglion cells at the distal part of the eighth auditory nerve, the wave II from the globular cells in the cochlear nucleus, the wave III is generated by the cochlear nucleus spherical cells and globular cells, and wave IV and wave $\mathrm{V}$ are generated from the medial superior olive and its projections to the nuclei in the lateral lemniscus and the inferior colliculus [56]. These electrophysiological responses are typically less than a microvolt in amplitude $[57,58]$. AMLRs are typically composed of a set of positive (P-waves) and negative ( $\mathrm{N}$-waves) waves. AMLRs are sensitive potentials for the processing of lowfrequency tones. The difference found between the behavioral auditory threshold and the electrophysiological threshold is of approximately $10 \mathrm{~dB}$ [59]. Further, they may be used for the investigation of functional integrity in the auditory pathway or the assessment of nonorganic hearing loss. Both measures will be recorded with four external electrodes placed over the forehead and behind the ear during the presentation of standardized acoustic signals while patients are lying in a quiet room. Measurements will be performed by trained medical and/ or study staff. These measurements will not be used as outcome measures, but will be analyzed as potential prognostic factors for treatment response.

\section{Audiometric and tinnitometric measures}

Otological examinations will be executed according to state-of-the-art medical practice. Audiological and/ or tinnitometric assessments will be conducted by responsible medical and/ or trained study staff with a standard clinical audiometer. Pure tone air-conduction and boneconduction audiometry will be recorded according to the guidelines from the British Society of Audiology http://www.thebsa.org.uk/wp-content/uploads/2014/04/ BSA_RP_PTA_FINAL_24Sept11_MinorAmend06Feb12. pdf. Individual tinnitus characteristics such as pitch and loudness will be determined via the presentation of different frequencies (or narrow band noise in the case of noise-like tinnitus). Participants have to decide which stimulus is closer to their tinnitus percept in a forced choice paradigm. Two different pure tones with a difference of two octaves $(1 \mathrm{kHz}$ and $4 \mathrm{kHz})$ will be consecutively presented and patients have to select the frequency which is closer to their tinnitus pitch (not intensity). Next, the selected frequency plus a different frequency closer to the one selected (one octave difference) will be presented and patients have to make the same decision again. The procedure goes on, keeping the closest frequency for the next round, until patients confirm the same frequency three times. In each round, different neighboring frequencies are presented. Frequencies used include 250, 500, 1000, 2000, 3000, 4000, 6000, and $8000 \mathrm{~Hz}$, presented at $20 \mathrm{~dB}$ above the individual hearing level of the respective frequency.

Subsequently, tinnitus loudness is determined by the comparison of the determined tinnitus frequency at different loudness levels starting from the individual hearing level ( $5 \mathrm{~dB}$ steps).

The procedures are conducted in one ear in case of unilateral (contralateral to tinnitus percept) or symmetric bilateral tinnitus and in two ears in cases of asymmetric tinnitus.

In order to assess patients' minimum masking level, a narrow band noise centered at the previously defined tinnitus frequency is presented in an ascending way (5 $\mathrm{dB}$ steps, starting from individuals hearing threshold) ipsilateral to the tinnitus percept until an adequate level to cover the patients' tinnitus is reached.

The phenomenon of a brief tinnitus percept suppression following acoustic stimulation is termed residual inhibition [60] and will be evaluated via 30-s and 2-min presentations of a pure tone and a broad band noise in accordance with the determined individual tinnitus frequency.

\section{Blood samples}

Blood samples are taken once before treatment start on a voluntary basis and are therefore not mandatory for trial participation. Participants are informed about blood sample collection and how their samples will be handled in detail (with respect to EU and country-specific regulations). The blood samples are sent to the Center for Genomics and Oncological Research (GENyO), Universidad de Granada (Granada, Spain) and the plasma samples to the Karolinska Institutet (Stockholm, Sweden) for further analysis of genetic parameters and plasma proteins respectively. Both laboratories are part of the UNITI consortium [38]. Storage, shipping, and analysis of participants biological samples are done according to the EU GDPR (2016/679) as well as country-specific legislations.

Peripheral blood samples from patients included in the clinical trial will be obtained in EDTA coated tubes. After centrifugation at $1500 \mathrm{~g}$ during $10 \mathrm{~min}$, plasma and cellular fractions will be separated. Plasma will be immediately frozen at $-80^{\circ}$ to prevent protein degradation.

DNA isolation and genome sequencing DNA will be isolated from the buffy coat by the QIAamp DNA blood Mini kit (QIAGEN), according to the manufacturer's protocol. DNA quality will be assessed by absorbance measurements (Nanodrop 2000c, Thermo Scientific) and by $260 / 280$ and $230 / 260$ indexes to determine protein 
and salt content. DNA concentration will be determined by a fluorimetric method (Qubit) and Quant-iT technology (Invitrogen) and DNA integrity will be evaluated in $1 \%$ agarose gels and by Bioanalyzer technology [61].

For whole-genome sequencing, DNA libraries will be prepared by using the Truseq DNA PCR-free kit (350 bp). DNA will be sequenced in an Ilumina NovaSeq 6000 sequencing platform 150 paired-end reads $(150 \times$ $2 \mathrm{bp}$ ) with a throughput $30 \times(90 \mathrm{~Gb} / \mathrm{sample})$. Bioinformatic analysis will include alignment and mapping of raw data to the latest updated reference genome (GRCh38.p13), preprocessing of FASTQ and generation of BAM files, variant calling to obtain VCF files following GATK Best Practices guidelines, and annotation using Ensemble Variant Effect Predictor.

Analyzing the whole genome using next-generation sequencing delivers a base-by-base view of all genomic alterations, including single-nucleotide variants (SNV), small insertions and deletions, and large structural variations (copy number changes). Paired-end whole-genome sequencing involves sequencing both ends of a DNA fragment, which increases the likelihood of alignment to the reference and facilitates detection of genomic rearrangements, repetitive sequences, and gene fusions. Gene burden tests will be used to compare burden of loss of function SNV and structural variants in synaptic genes in patients with tinnitus, using Non-Finnish European dataset from gnomAD v3 as population database to estimate allelic frequencies [62].

Protein analyses Protein biomarkers for patients included in the RCT will be initially assessed by a proximity extension assay (PEA) from O'LINK. Control groups will include subjects with and without hearing loss. PEA multiplex analysis in 500 tinnitus cases and 2728 non tinnitus control blood samples from the STOP study (https://stop.ki.se/) will be performed. An immune response biomarker panel directed against 96 proteins implicated in inflammatory diseases will be used, since several studies have found inflammation to be involved in various forms of hearing loss. Therefore, inflammatory biomarkers could potentially be associated with tinnitus. Standard biostatistical analyses will be performed, including basic descriptive and univariate statistics as well as receiver operating characteristics curves.

Genetic and blood biomarkers will serve as a basis for determining tinnitus subtypes and evaluating persons' treatment response at the clinical sites through the procedure.

\section{Treatment conditions}

Four different types of interventions will be included in the present RCT. Two of them mainly target on the auditory aspects of tinnitus (sound therapy; hearing aid), whereas the other two especially target the CNS (structured counseling; cognitive behavioral therapy). Each intervention will be harmonized among the participating clinical sites in view of procedure, technical equipment, and trained research staff. This will be achieved via specific Standard Operation Procedure documents and a detailed step-by-step description of procedures as well as the conduction of dedicated workshops from leading experts in the particular field for each intervention. Hereinafter, the used interventions are described.

\section{Sound therapy (ST)}

Sound therapy will be self-administered on a daily basis and conducted via a dedicated mobile application for Android and iOS in accordance to the European regulations for medical software (IEC 62304, IEC 82304). This mobile application will contain a set of 64 different stimuli comprised of various artificial and naturalistic sounds and an application of different state-of-the-art filter or modulation techniques, e.g., amplitude modulated tones or noises [63, 64] as well as filtered music [65]. Intensity and length of sound stimulation can be adjusted by the user. Maximum loudness for all types of sound stimulation will be $85 \mathrm{~dB}$. Patients' behavioral data (tinnitus loudness after stimulation) as well as type of sound, loudness, and play duration/ repetitions will be collected for each conducted stimulation. Further, the time of app usage will be recorded. All personal data will be treated with the upmost confidentiality and multiple efforts will be made in order to pseudo-anonymize the collected data and to protect participants' identities. EU General Data Protection Regulation (GDPR, 2016/679) will apply for the mobile application, which are outlined in the data management plan in the supplemental material.

\section{Hearing aid $(H A)$}

Participants will be stratified according to their hearing function. Patients with a hearing aid indication will represent one strata and only these patients will be randomized for the option "hearing aid therapy." Conventional commercially available hearing instruments will be utilized with all tinnitus-specific sound interventions deactivated, in order to disentangle the effect of amplification from the effects of sound therapy. Specifically, all participants were fitted bilaterally with mini-behind-the-ear hearing instruments (Type Signia Pure 312 7X; Sivantos Pte. Ltd., Singapora, Republic of Singapore/ WSAudiology, Lynge, Denmark), with acoustic coupling achieved via non-custom, non-occluding eartips. Gain assignments appropriate for each individual's hearing loss will be applied according to the National Acoustic Laboratories-Non-Linear 2 generic amplification prescription procedure [66] and verified using probe microphone measures. As it has been reported that numerous 
signal processing characteristics might significantly affect the impact of amplification on tinnitus, all signal processing features, excepting only acoustic feedback reduction and impulse noise reduction algorithms, will be deactivated during the 12-week hearing aid trial. Verification of hearing aid gain assignments and signal processing will be performed by audiologists, hearing aid acousticians, or trained study personnel, in accordance with the appropriate practice guideline of the British Society of Audiology (http://www.thebsa.org.uk/ wpcontent/uploads/2018/05/REMS-2018.pdf). Participants will be encouraged to use their hearing aids for at least $10 \mathrm{~h}$ per day and will be allowed to keep their hearing devices following successful completion of the RCT.

\section{Structured counseling (SC)}

A standardized protocol developed from a team of psychologists will provide structured patient education, counseling, and tips for a life with tinnitus on a daily basis. The protocol is oriented towards recent European Tinnitus Guidelines [17] and comprised of 12 chapters:

(1) Basic facts about tinnitus

(2) Beginner facts about tinnitus

(3) Beginner facts about the brain and sound perception

(4) Important vocabulary

(5) Myths and misconceptions about tinnitus

(6) Diagnosis of tinnitus

(7) Special types of tinnitus

(8) Therapeutic approaches

(9) Psychology of tinnitus

(10)My behavior and my tinnitus

(11)The science on tinnitus

(12)Switch perspective: How would you advice others?

Structured counseling will be equally implemented via a mobile application on Android and iOS in compliance with the European regulations for medical software (IEC 62304, IEC 82304) - details can be found in the data management plan in the supplemental material. At the end of each chapter, there will be a short quiz to repeat and consolidate the content. Answers and scores of the quizzes as well as time of app usage will be collected according to the General Data Protection Regulation (GDPR, 2016/679). Patients will be encouraged to use the specifically developed app for structured counseling. However, alternatively the information contained in the app can also be provided in printed form.

\section{Cognitive behavioral therapy for tinnitus (CBT4T)}

A specially designed cognitive behavioral therapy (CBT) program for tinnitus patients will be applied by psychologist or psychotherapists by training. CBT4T will be performed in weekly face-to-face group meetings á 1.5-2 $\mathrm{h}$ over the 12-week treatment phase. One face-to-face screening visit will take place before the group session. The maximum number of participants per group will be six tinnitus patients. Psychologists and psychotherapists will carry out the CBT4T and are trained by workshops before the treatment. The theoretical concept and the content of the treatment of CBT4T is based on the concept of exposure therapy in anxiety disorders or chronic pain (cognitive restructuring via exposition to fear expectations) and a fear-avoidance model [18, 67, 68]. Short psychoeducation at the beginning of the treatment is followed by exposure exercises in group sessions.

\section{Trial procedure}

The UNITI-RCT will be conducted at the five clinical sites as well as in patients' homes. All patients have to make at least four visits in the respective clinical sites over a period of at least 10 months consisting of screening, baseline visit, interim assessment, final visit at treatment end, and a follow-up visit 36 weeks after the baseline assessment. Patients who will be randomly assigned to CBT4T have to additionally visit the respective clinical site twelve times in order to participate in the CBT sessions. All other treatments are conducted without local restrictions.

Screening, baseline, and treatment start can be performed on the same day. In this case, relevant assessments are only performed once. Furthermore, an additional visit in the screening period can be added for purposes of screening/ baseline procedures. Prior to the first screening visit, there will be an online pre-screening in order to preselect potentially appropriate participants.

The interval between baseline and treatment start should be maximally 4 weeks. If the interval is longer, an additional visit will be done (without ESIT-SQ, TSCHQ, BFI-2, ATAQ, electrophysiological measurements, or otological examination). Blood samples will be taken before treatment start. Consent for the participation at the genetic and plasma protein tests is independent from the consent to participation in the clinical trial. In case of a combinational treatment, the second treatment will start maximum ten working days after the first (e.g., HA and CBT4T). Moreover, a facultative additional followup assessment 48 weeks after baseline measurements is planned. If a patient drops out for any reason or wishes to discontinue the treatment prematurely, there is the possibility to continue with study visit participation.

For each study visit, a deviation of \pm 7 days is permitted. Figure 1 gives an overview of the study procedure, while in Table 2 the assessments for all measurement points are outlined. In the following sections, each visit is shortly described in a chronological order. 


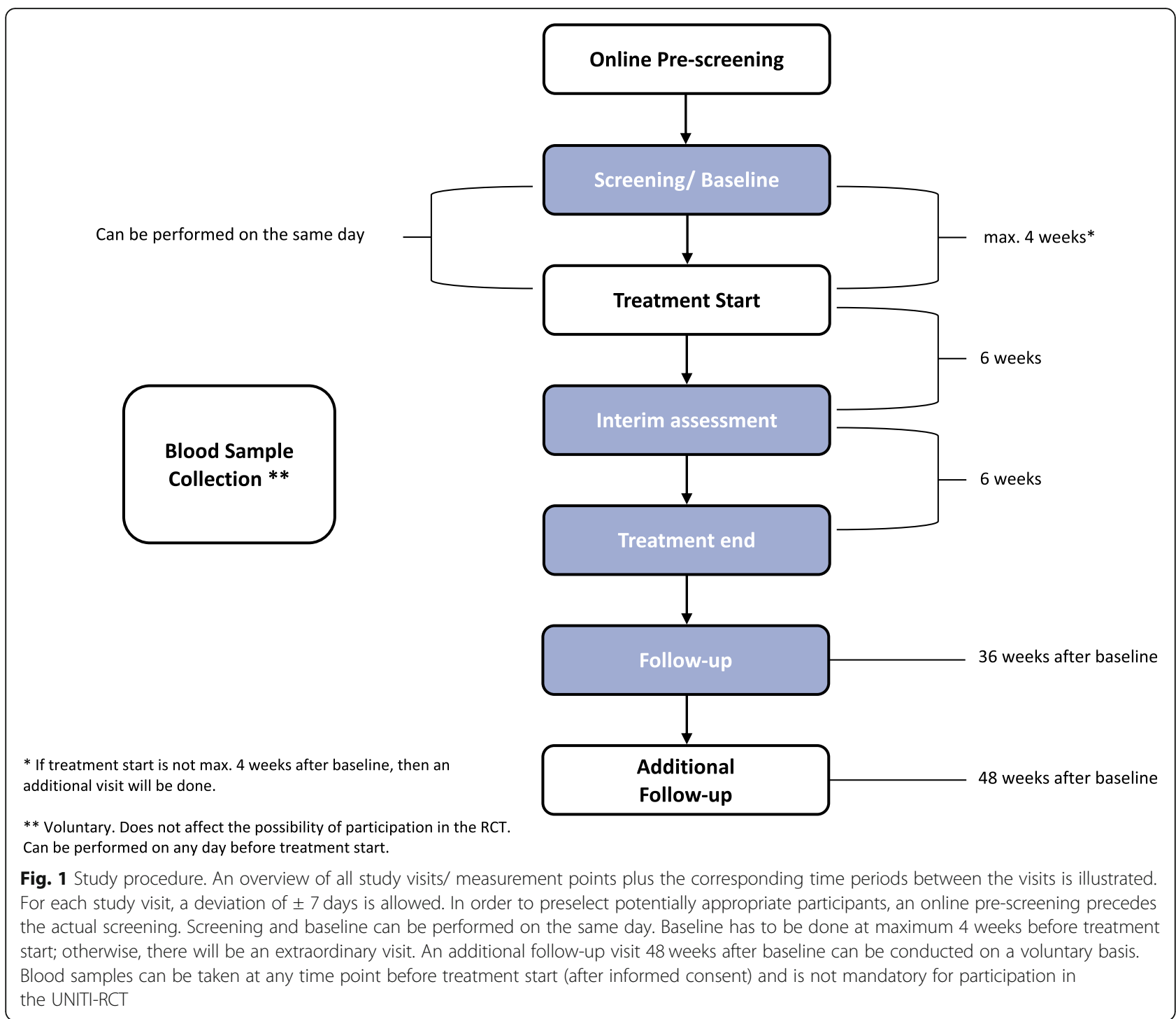

\section{Pre-screening}

Due to the investigation of a rather large and specific sample of the tinnitus population, a pre-screening will precede the actual screening process. This prescreening is conducted as an additional layer of the eligibility assessment in the form of an online-based survey and designed to ensure a more efficient eligibility check (for an overview of study eligibility criteria see Table 1). Access to the pre-screening survey will be provided via a URL link to interested candidates. Before start of the pre-screening, candidates will be provided with information about study aim, procedure, and participation requirements. Hereinafter, participants have to confirm that they read and understood the provided information plus that they are willing to participate in the pre-screening process and answer a series of tinnitus- and health-associated questions. After completion of the pre-screening, patients will get a six-digit code, have to contact the respective study center, and leave their patient-code and phone number. Within 7 days, patients will get their results about eligibility and, if applicable, information about the subsequent screening assessment. Eligible candidates who accept the screening assessment invitation will be sent a Patient Information Leaflet and Informed Consent Form (ICF) by regular mail, to assure they have sufficient time to read the provided information and formulate questions ahead of the screening visit. Candidates who are not eligible to participate in the study will receive suggestions for treatment options outside the UNITI study.

\section{Screening}

Prior to start of the screening, participants will be informed about purpose, procedure, and potential risks or benefits of the trial by a member of the local study team 
Table.2 Schedule of assessments

\begin{tabular}{|c|c|c|c|c|c|c|c|c|}
\hline & Pre-screening & Screening & Baseline & $\begin{array}{l}\text { Treatment } \\
\text { start }\end{array}$ & $\begin{array}{l}\text { Interim } \\
\text { visit }\end{array}$ & $\begin{array}{l}\text { Final visit }=\text { end } \\
\text { of treatment }\end{array}$ & Follow-up & $\begin{array}{l}\text { Additional } \\
\text { follow-up }\end{array}$ \\
\hline ICF & $A^{a}$ & A & & & & & & \\
\hline Eligibility criteria & A & A & A & & & & & \\
\hline ESIT-SQ & & & A & & & & & \\
\hline TSCHQ & & & B & & & & & \\
\hline Mini TQ & A & A & A & & A & A & A & B \\
\hline Tinnitus numeric rating scales & & A & A & & A & A & A & B \\
\hline TFI & & A & A & & A & A & A & B \\
\hline THI & A & A & A & & A & A & A & B \\
\hline WhoQol-BREF & & A & A & & A & A & A & B \\
\hline BFI-2 & & & A & & & & & \\
\hline CGI-I & & & & & A & A & A & B \\
\hline GUF & & B & B & & B & B & B & B \\
\hline PHQ-D & A & A & A & & A & A & A & B \\
\hline Mini-SOISES & & & A & & A & A & A & B \\
\hline ATAQ & & & $B^{b}$ & & & $B^{b}$ & & \\
\hline FTQ & & & B & & B & B & B & B \\
\hline MoCA & & A & & & & & & \\
\hline Randomization & & & A & & & & & \\
\hline Blood sampling & & & $B^{c}$ & & & & & \\
\hline Otological examination & & A & & & & A & B & B \\
\hline Audiometry & & A & & & & A & B & B \\
\hline Loudness match & & A & & & & A & B & B \\
\hline Pitch match & & A & & & & A & B & B \\
\hline Maskability & & A & & & & A & B & B \\
\hline Residual inhibition & & A & & & & B & B & B \\
\hline ABR & & & A & & & & B & B \\
\hline AMLR & & & A & & & & B & B \\
\hline Treatment & & & & A & A & A & & \\
\hline Comorbidities & & A & A & A & A & A & A & B \\
\hline Concomitant medication/ treatment & & A & A & A & A & A & A & B \\
\hline Adverse events & & & & & A & A & A & B \\
\hline
\end{tabular}

A mandatory; B voluntary; ICF Informed Consent Form; ESIT-SQ European School of Interdisciplinary Tinnitus Research Screening Questionnaire; TSCHQ Tinnitus Sample Case History; Mini-TQ Mini Tinnitus Questionnaire; TFI Tinnitus Functional Index; THI Tinnitus Handicap Inventory; WhoQol-BREF World Health Organization Quality of Life - abbreviated; BFI-2 Big Five Inventory-2; CGI-I Clinical Global Impression Scale - Improvement; GUF Questionnaire on Hypersensitivity to Sound; PHQ-D Patient Health Questionnaire for Depression; SOISES Social Isolation Electronic Survey; ATAQ Attitudes Towards Amplification Questionnaire; FTQ Fear of Tinnitus Questionnaire; MoCA Montreal Cognitive Assessment; ABR Auditory Brainstem Response; AMLR Auditory Middle Latency Response

Screening and Baseline measurements as well as treatment start can be performed on the same day. In this case, all measurements are only performed once. The baseline should be maximum 4 weeks before the treatment start; otherwise, baseline measures should be repeated (without ESIT-SQ, TSCHQ, BFI-2, ATAQ, electrophysiological measurements). ${ }^{\mathrm{a}}$ Declaration of consent (ICF) can be digital for the pre-screening. ${ }^{\mathrm{b}}$ Only for participants who were allocated to a single or combinational treatment with $\mathrm{HA}$. 'Blood samples can be taken at any time point before treatment start

and have to give written informed consent. Consent for the participation in the genetic tests is independent from the consent to participation in the clinical trial. Informed consent forms plus information sheets for the RCT and the blood analysis can be found in the supplemental material. Screening will be conducted at the respective clinical sites. During screening, inclusion and exclusion criteria (cf. Table 1) are verified in a clinical face-to-face setting. Several exclusion criteria are checked once again to verify that there were no changes as compared to prescreening. In the course of the screening process, participants undergo a set of otological, audiometric, and 
tinnitometric examinations and have to complete several tinnitus- and health-related questionnaires. In addition, their cognitive abilities will be assessed with the MoCA test [52].

\section{Baseline}

After a successful screening visit and a signed ICF, several baseline measures consisting of tinnitus- and healthrelated questionnaires and electrophysiological measurements are conducted. Following baseline measurements, participants are randomly allocated to a treatment group (please see section "Stratification and randomization" below) and informed about the specific treatment they receive.

\section{Stratification and randomization}

Participants will be randomly assigned to one treatment arm comprised of a single or combinational type of intervention as exemplified in Fig. 2. Stratification will be conducted on the basis of two criteria determined during screening. As a first step, participants will be stratified according to the degree of tinnitus severity (Fig. 2, step 1). Based on their THI score [41], participants will be allocated to a low (score $<48$ ) or a high (score $\geq 48$ ) tinnitus distress group. Subsequently, the decision will be made, whether there is an indication for the application of a HA. Accordingly, the two subgroups of low and high tinnitus distress participants will be stratified into two groups as indicated in step 2 of Fig. 2: participants with an HA indication and those without an indication. If eligible participants have already worn a HA 3 months before screening, they will be automatically allocated to the stratification group of no HA indication. An equal ratio between the four strata (HA yes, $\mathrm{THI} \geq 48$; HA no, THI $\geq 48$; HA yes, THI $<48$; HA no, $\mathrm{THI}<48$ ) is intended. In a final step, participants will be randomly assigned to one intervention arm (cf. Fig. 2, step 3 ). The randomization will be executed per clinical

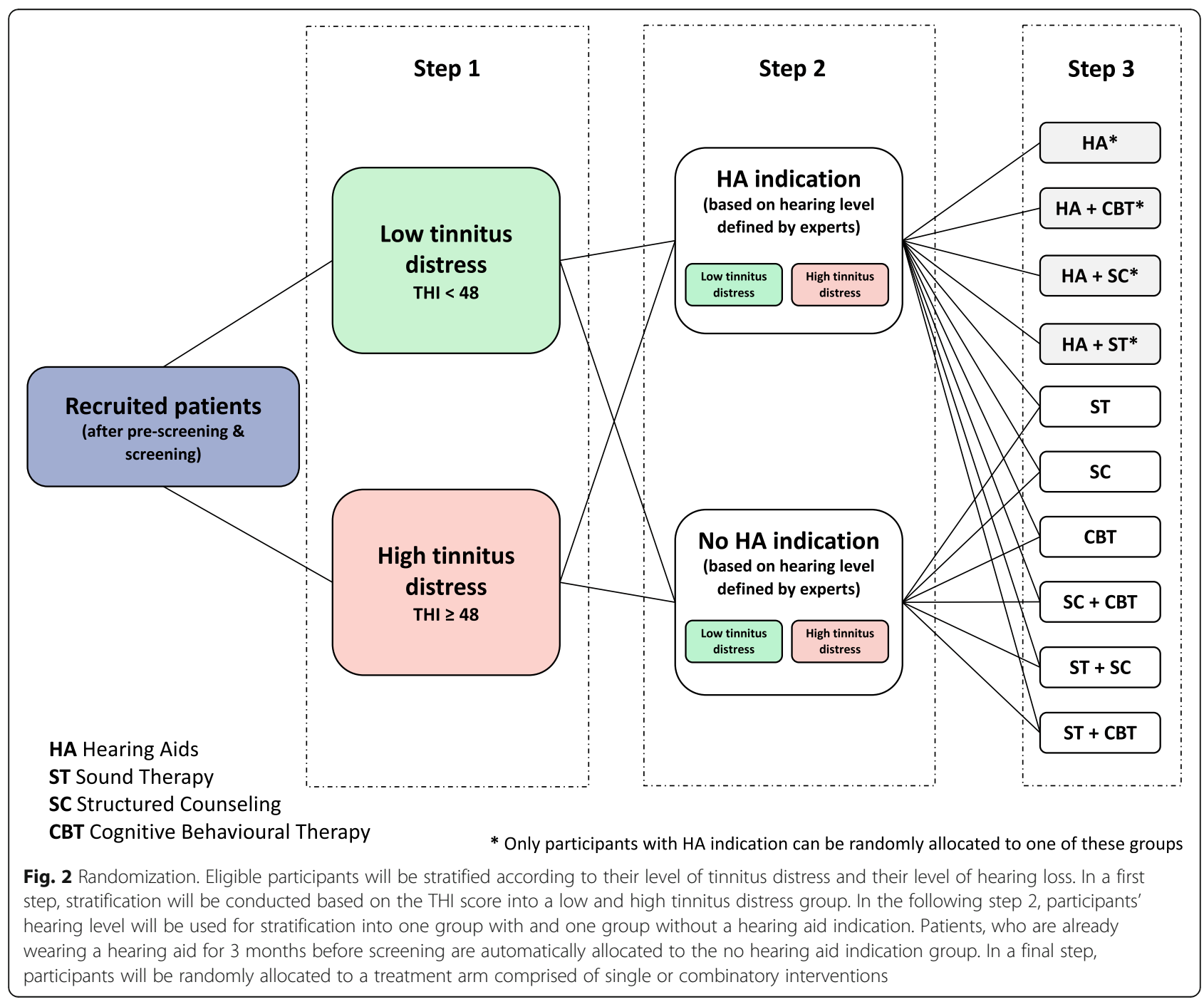


site and monitored centrally (see section "Data management and study monitoring"). A specific interactive web response system (IWRS) will help investigators at the clinical sites to randomize their patients. This facilitates the management of a large number of patients from different sites located in several countries and the monitoring of the multicentric study with a complex design. The distribution across the four strata will be centrally monitored during the randomization process. If a recruited and eligible participant quits the RCT participation before randomization, this participant will be considered as a screening failure. In case an eligible participant is already randomized to a treatment group and quits study participation, this patient will be considered as a drop-out. The aim is to have 100 patients randomly allocated to a treatment group per clinical center.

\section{Interim assessment}

After 6 weeks of treatment, participants are required to make one interim visit at the clinical site in order to complete several health- and tinnitus-related questionnaires. Comorbidities, concomitant medication, and treatments as well as potential adverse events are recorded. In case of adverse events directly related to the treatment, the responsible principle investigator has to make a decision about discontinuation of the intervention. This can happen at any time during the treatment phase, since participants will be instructed to get in contact with the staff of the respective clinical site in case of adverse events. This interim visit can also be conducted online via the UNITI database in order to reduce clinical visits. Patients will get a URL link to fill out the corresponding questionnaires. Comorbidities, concomitant medication, and treatments as well as potential adverse events will be assessed telephone call.

\section{Final assessment/ end of treatment}

After 12 weeks, the treatment phase ends. Health- and tinnitus-related questionnaires are filled out, and several audiometric and tinnitometric measurements are executed.

\section{Follow-up assessments}

The first follow-up visit will take place 36 weeks after the baseline visit and consists of the same measures as taken at the final assessment/end of treatment. Though, the implementation of all otological, audiological, or electrophysiological measures are not mandatory and can be decided by the clinical sites individually. An additional facultative follow-up takes place 48 weeks after the baseline visit. Same measurements are conducted as during the first follow-up.

\section{Data management and study monitoring}

The contract research organization Excelya (www. excelya.com) will monitor the whole trial and will perform on-site visits in order to assess the progress of the study and to ensure that it is conducted, recorded, and reported in accordance with the protocol, the timelines, Good Clinical Practice, and any other applicable regulations.

Data collected at the clinical sites will be entered in the UNITI database and treated as securely as possible and protected according to the latest international guidelines. In terms of data protection, personal data will be managed according to the EU guidelines. Participants will receive a pseudo-anonymized code, which will be used during the course of the study. Personal data will be stored at the respective clinical sites and not entered in the UNITI database. General Data Protection Regulations (GDPR) from the EU apply for all UNITI mobile applications. Full details of data protection and data security can be found in the accompanying UNITI data management plan (see supplementary material). Any disclosure of study data for scientific purposes will only be made in anonymous form.

\section{Adverse events}

Adverse events (AE) and serious adverse events (SAE) are defined according to Good Clinical Practice $\$ 3(6,8)$. Every AE during the study will be documented with respect to start/ finish date, intensity, relation to intervention, consequences for intervention, and what actions are taken (e.g., hospitalization). Any SAE occurring during the treatment phase will be recorded and reported to the local ethics committee and the coordinator of the trial within $24 \mathrm{~h}$. If a patient reports an SAE, the intervention will be immediately stopped. The study coordinator together with the local principal investigator of the respective center will decide if the participant should cease the specific treatment and which further measures are taken.

\section{Risks associated with participation}

There is a minor risk that the interventions used could lead to an increase of subjective tinnitus symptoms like loudness or unpleasantness. From clinical experiences, temporal increases of symptoms occur in $10 \%$ of cases. In case of tinnitus deterioration or any other adverse event, participants can quit the intervention without giving reason at any time. If desired they can receive support by trained medical or psychological experts at the specific clinical site.

Since participants have to visit clinical sites several times (e.g., study visits or CBT4T groups), there might be a slightly increased risk for a COVID-19 infection. However, safety precautions at the clinical sites following 
all country-specific regulations and the fact that the majority of the interventions can be performed at patient's home (HA and mobile applications) keep the risk very low; nevertheless, it cannot be excluded. Conduction of the study will be done according to the regulations of the present local hygiene plans at the respective clinical sites. As all offered treatments are well established and as their safety profile is well known, the potential benefits of participating in this study outweigh the potential risks like a short-term increase of subjective tinnitus symptoms.

\section{Statistical analysis}

The statistical analysis plan for the study data will be finalized before all data is collected as well as before the closure of the UNITI database and will be published separately. Hence, the following statistical analysis section provides only a brief excerpt.

Statistical analysis will be performed by a dedicated data analysis team blinded to the type of intervention. This will be accomplished by pseudo-anonymizing the treatment codes, which will only be available to the center-specific investigator responsible for the randomization. The final sample size was estimated through power calculation as already outlined above, to address the main contrast of interest, that is, whether there is a statistically significant difference in the primary outcome when comparing single and combinatory intervention. Several layers of analysis will be applied, on top of descriptive statistics. As primary outcome, tinnitus improvement (change in THI score) will be compared between the single treatment and the combinational treatment arms. Each one of the outcome measures will be compared between groups, in terms of difference between baseline and end of treatment respectively final follow-up visit. On top of this, all different groups will be compared among each other. Aim of this comparison is to identify differences between each one of the groups separately. Outcome measures will be handled as previously described. In addition, patients who underwent one specific treatment, either as single or part of combinational therapy will be grouped together, formulating four groups, representing the four different interventions (ST, HAs, CBT4T, and SC) and analyzed accordingly. This analysis will estimate the effectiveness of each one of the interventions, regardless if they were provided alone or in combination with any other treatment. Furthermore, a comparison of the subgroup of patients who received an intervention alone versus the subgroup representing those who underwent the same therapy in combination with any other kind of therapy will be conducted. For example, patients of the single treatment arm, who only received HA as intervention, will be compared with patients from the combinational arm who received HA and any other treatment and so forth. Tinnitus improvement will be further compared between ear-mediated (HA, ST) and brain-mediated (CBT4T, SC) types of interventions.

Appropriate statistical methods will be applied ( $t$-test, ANOVA, etc.) under the supervision of biostatisticians. Descriptive statistics will be used for the primary and secondary outcomes, as well as included covariates. Categorical variables will be compared with chi-square tests, whereas repeated measures (pre and post treatment) will be compared with paired $t$-tests or repeated measure ANOVAs, in the case of more than two groups, after testing for distributional assumptions. Non-parametric tests will be used if the assumptions of parametric tests, such as equal variance between groups and Gaussian distributions, are not met. These tests will be used to evaluate the primary outcome across timepoints (e.g., baseline, interim visit, final visit, follow-up visit). Regression strategies with random-effect models will also be applied in order to evaluate which of the factors were determinative for each one of the outcome measures. To this end, socio-demographics, as well as questionnaires quantifying depression and tinnitus-related distress collected at baseline, will be used as covariates. Several hierarchical models will be created using the lme4 package in R. The main goal will be to identify which baseline predictors are associated with the THI score by the end of treatment. The first set of models will compare treatments with and without hearing aids (CBT vs. CBT $+\mathrm{HA}, \mathrm{ST}$ vs. ST + HA, SC vs. SC + HAs). The second set of models will assess the effect of baseline predictors on the THI by the end of the treatment in single and combinatorial treatments $(\mathrm{SC}+\mathrm{CBT}, \mathrm{ST}+\mathrm{SC}, \mathrm{ST}+$ CBT). Next, individual treatments will be compared against each other (CBT vs. HA vs. ST vs. SC). Finally, treatments will be divided into ear-mediated (e.g., HA and ST) and brain-mediated (e.g., CBT and SC). All models will have each center encoded as a random factor. Models will be adjusted with the covariates as described above. These analyses will aim to find the important parameters for the course in each one of the domains encountered by each one of the outcome measures. Significance level will be set to $5 \%$ for all analyses. Another objective of this RCT is the validation of a specific Decision Support System (DSS) per patient. To this end, all cases will be grouped post hoc based on whether the treatment they received based on randomization happened to be identical or not to that suggested by the DSS according to their profile. The two groups will be named DSS aligned (+) and DSS aligned (-). Whether a patient enters one of the two groups will be a matter of coincidence and not subject to randomization or actual timely DSS suggestion, since this system will not be ready for the RCT start. Evaluation of the DSS will be 
twofold. Initially, the outcome between DSS aligned (+) and DSS aligned (-) groups will be compared with use of the outcome measures used in the RCT. Secondly, the proportion of patients with clinically significant improvement will be compared between the two groups, indicating the positive and negative prognostic values of the platform respectively.

\section{Ethics/ ethical aspects}

The study procedure has been submitted to and approved by the local ethics committees responsible for research activities at all investigator clinical sites. Positive evaluated ethic votes for the clinical sites in Granada, Athens, Berlin, Regensburg, and Leuven (combined ethics approval for clinical sites in Germany) can be found in the supplemental material. Prior to start of the study eligible patients have to give written informed consent. Each patient will get detailed and comprehensive information about the objectives, procedures, and interventions of the RCT plus potential side effects associated with a participation as well as their right to cancel a participation at any time during the study without any reason or detriment. Participants can request that their study data are removed from the database and excluded from future analysis. All documentation that is needed for possible regulatory audits, e.g., the signed ICF, cannot be deleted, even if this is requested by the patient. Any changes required to the protocol are discussed and decided jointly in a dedicated meeting of the responsible PIs. All amendments will be prepared by the RCT coordinator team in Regensburg and submitted to all local ethics committees.

\section{Discussion}

The present study protocol describes an international multi-center RCT for chronic tinnitus using four different types of interventions (HA, ST, SC, CBT4T), performed either as a single treatment or a combination of two treatments over the course of 12 weeks.

For many people affected, tinnitus constitutes a massive burden with many potential psychological and physiological comorbidities $[1,69,70]$, ultimately resulting in lower quality of life $[71,72]$. The used interventions in the UNITI-RCT might have the potential for brief or even prolonged tinnitus suppression as well as to help people to better cope respectively live with their tinnitus.

With its primary objective to evaluate not only single treatments but also the combination among them, the UNITI-RCT will be the first proper systematic RCT in tinnitus to scrutinize single and combinatory interventions in more depth. Given the fact that numerous single-target studies have failed to establish an effective therapy, the combination of different types of interventions allows a targeting of different involved organ levels at the same time (AS, CNS) and might manifest in better treatment responses.

Furthermore, the UNITI-RCT attempts to overcome essential limitations of previous studies $[20,21]$ through a large sample size, harmonized patient selection and screening processes across the five participating clinical centers, standardized assessments methods, and interventions across the clinical sites, and large-scale data analysis strategies. Previous reviews of tinnitus treatments provide evidence that only three past RCTs exceed 250 participants [23]; to the best of our knowledge, the currently largest RCT included 492 patients [18]. With the aim to investigate 500 tinnitus patients, the UNITI-RCT has the attempt to be the largest RCT in tinnitus yet. Importantly, with the UNITI-RCT, we also include the combination of treatments.

Due to tinnitus heterogeneity [24] as well the lack of clarity about pathophysiological processes [73, 74], upto-now there is no cure for tinnitus [3, 75]. In the majority of studies, only a subgroup of tinnitus patients demonstrates improvement [23]. It has been proposed that precision medicine approaches should be tackled by future tinnitus research [21, 24]. The UNITI-RCT aims at making a significant contribution to the personalization of tinnitus treatments by means of the validation of a specific DSS. The system will be developed during the UNITI project [38], in order to support clinicians in choosing the optimal treatment based on an individual patient's profile [39]. Such a system and its underlying computational model has not only the potential to significantly contribute to our understanding of the tinnitus pathology, but also improve clinical practice and the way we are trying to treat tinnitus.

It has already been suggested that mobile applications offer a promising way for tinnitus assessment and treatment [76-78]. Hence, several specific mobile applications will be developed for the UNITI-RCT creating the opportunity to significantly reduce the number of tinnitus-related clinical visits and as such the necessary health care resources. Moreover, these mobile applications do not only provide an ecological way to evaluate patients' tinnitus at many different timepoints with the possibility to track daily tinnitus fluctuations and detailed treatment-related consequences, but also provide maximum flexibility in space and time from a patients' view-potentially increasing compliance.

Taken together, the UNIT-RCT with its multidisciplinary multi-center approach, standardized state-of-theart tinnitus interventions and assessments, novel treatment combinations, and mobile applications as well as its major contribution to personalize tinnitus treatments might not only represent a new methodological benchmark in tinnitus trials but also provide an essential step towards a cure for tinnitus. 


\section{Status of the trial}

Issue date: 04. November 2021. Protocol version no. 3. The first stage of patient recruitment started in March 2021 with the online pre-screening. In April 2021, screening and baseline measurements started at the clinical sites in Berlin and Regensburg. In June 2021, these measurements started at the clinical site in Granada and Athens as well. The trial including all mandatory assessments at all involved clinical sites is due to be finished by the end 2022 .

\begin{abstract}
Abbreviations
ABR: Auditory brain stem response; AE: Adverse event; AMLR: Auditory middle latency responses; ANOVA: Analysis of variance; AS: Auditory system; ATAQ: Attitudes Towards Amplification Questionnaire; BFI-2: Big Five Inventory-2; CBT4T: Cognitive behavioral therapy for tinnitus; CGI-I: Clinical Global Impression Scale - Improvement; CNS: Central nervous system; DNA: Deoxyribonucleic acid; DSS: Decision support system; ESITSQ: European School of Interdisciplinary Tinnitus Research Screening Questionnaire; EU: European Union; FTQ: Fear of Tinnitus Questionnaire; GDPR: General Data Protection Regulations; GUF: Questionnaire on Hypersensitivity to Sound; HA: Hearing aid; ICF: Informed consent form; MiniTQ: Mini Tinnitus Questionnaire; MoCA: Montreal Cognitive Assessment; PEA: Proximity extension assay; PHQ-D: Patient Health Questionnaire for Depression; RCT: Randomized clinical trial; SAE: Serious adverse event; SC: Structured counseling; SNV: Single-nucleotide variants; SOISES: Social Isolation Electronic Survey; ST: Sound therapy; TFI: Tinnitus Functional Index; THI: Tinnitus Handicap Inventory; TSCHQ: Tinnitus Sample Case History Questionnaire; UNITI: Unification of Treatments and Interventions for Tinnitus Patients; WhoQol-BREF: World Health Organization Quality of Life abbreviated
\end{abstract}

\section{Supplementary Information}

The online version contains supplementary material available at https://doi. org/10.1186/s13063-021-05835-z.

Additional file 1. Ethical approvals from Germany, Spain, Greece and Belgium. Informed consent form - RCT. Information sheet - RCT. Informed consent form - blood sampling. Information sheet - blood sampling. UNITI data management plan. WHO trial registration dataset.

\section{Authors' contributions}

The authors BL, WS, MS, BM, JL-E, DK, RC, and SS (Stefan Schoisswohl) are main responsible for the conceptualization of the study. Author SS (Stefan Schoisswohl) wrote the main manuscript together with WS, MS, and BL. Methodological procedures were written together with corresponding experts of the UNITI consortium: SM (hearing aid fitting); PN, WS (sound therapy); MS (cognitive behavioral therapy); MS, WS (structured counseling); SM, DK (audiometry and measures of tinnitus characteristics); JS, DK (data analysis), JL-E (blood sampling); DK (electrophysiological measurements). All authors read and approved the final manuscript.

\section{Funding}

The UNITI project has received funding from the European Union's Horizon 2020 Research and Innovation Program (grant agreement number 848261).

\section{Availability of data and materials}

Not applicable

\section{Declarations}

\section{Ethics approval and consent to participate}

As already mentioned, the study procedure has already been positively evaluated by the local ethics committees in Granada, Athens, Berlin, Leuven, and Regensburg. The ethical approval documents can be found in the supplemental material. Prior to study start/ blood sampling, all participants have to give informed consent. RCT and blood sampling information sheets and consent forms are available from the supplemental material.

\section{Consent for publication}

Not applicable

\section{Competing interests}

The authors declare that they have no conflict of interest associated with this publication and there has been no significant financial support that could influence the conduction of the RCT. The used hearing aid devices are provided by Sivantos Pte. Ltd/ WSAudiology. The devices for the measurement of acoustic evoked potentials are provided by Interacoustic A/ $\mathrm{S}$. There is no influence of any company or authority on the design, conduction, analysis, or any other details of this RCT. One employee of Sivantos Pte. Ltd/ WSAudiology is part of the External Advisory Board of the UNITI project [38], but has no influence on the conduction of the RCT. The results of the study are only made available to companies or external authorities via peer-reviewed publications.

\section{Author details}

${ }^{1}$ Department of Psychiatry and Psychotherapy, University of Regensburg, Universitaetsstraße 84, 93053 Regensburg, Germany. ${ }^{2}$ Department of Otolaryngology, Instituto de Investigacion Biosanitaria Ibs.GRANADA, Hospital Universitario Virgen de las Nieves, Granada, Spain. ${ }^{3}$ Tinnitus Center, CharitéUniversitätsmedizin Berlin, corporate member of Freie Universität Berlin und Humboldt Universität Berlin, Berlin, Germany. ${ }^{4}$ Department of Physiology and Pharmacology, Karolinska Institutet, Stockholm, Sweden. ${ }^{5}$ EXCELYA Hungary Kft., Budakalász, Hungary. 'Department of Health Psychology, Katholieke Universiteit Leuven, Leuven, Belgium. ${ }^{7}$ Department of Neurosciences, Research group Experimental Oto-Rhino-Laryngology, University of Leuven, Leuven, Belgium. ${ }^{8}$ Department of Otorhinolaryngology - Head and Neck surgery, University Hospitals Leuven, Leuven, Belgium. ${ }^{9}$ Multidisciplinary University Center for Speech-Language Pathology and Audiology, University Hospitals Leuven, Leuven, Belgium. ${ }^{10}$ Terzo-Institute for Applied Hearing Research, ISMA, Sonneberg, Germany. ${ }^{11}$ Otology \& Neurotology Group CTS 495, Department of Genomic Medicine, GENYO, Center for Genomics and Oncological Research: Pfizer/University of Granada/Andalusian Regional Government, PTS Granada, Granada, Spain. ${ }^{12}$ Department of Mental Health, Hospital Universitario Virgen de las Nieves, Granada, Spain. ${ }^{13}$ Central Biobank Charité, Charité- Universitätsmedizin Berlin, corporate member of Freie Universität Berlin und Humboldt Universität Berlin, Berlin, Germany. ${ }^{14}$ Department of Otolaryngology, Head and Neck Surgery, National and Kapodistrian University of Athens, Hippocrateion General Hospital, Athens, Greece. ${ }^{15}$ Center for Clinical Studies, University Hospital Regensburg, Regensburg, Germany. ${ }^{16}$ Department of Surgery, Division of Otolaryngology, Universidad de Granada, Granada, Spain. ${ }^{17}$ Department of Otolaryngology, University Hospital Regensburg, Regensburg, Germany. ${ }^{18}$ Department of Otolaryngology, Instituto de Investigacion Biosanitaria ibs. GRANADA, Hospital Universitario Clinico San Cecilio, Granada, Spain. ${ }^{19}$ Center for Cognitive Neuroscience and Department of Psychology, University of Salzburg, Salzburg, Austria. ${ }^{20}$ Department of Psychosomatic and Psychotherapy, Charité- Universitätsmedizin Berlin, corporate member of Freie Universität Berlin und Humboldt Universität Berlin, Berlin, Germany.

Received: 28 May 2021 Accepted: 15 November 2021

Published online: 04 December 2021

\section{References}

1. De Ridder D, Schlee W, Vanneste S, Londero A, Weisz N, Kleinjung T, et al. Tinnitus and tinnitus disorder: theoretical and operational definitions (an international multidisciplinary proposal). Prog Brain Res. 2021;260:1-25. https://doi.org/10.1016/bs.pbr.2020.12.002.

2. Mazurek B, Olze H, Haupt H, Szczepek AJ. The more the worse: the grade of noise-induced hearing loss associates with the severity of tinnitus. Int J Environ Res Public Health. 2010;7(8):3071-9. https://doi.org/10.3390/ ijerph7083071.

3. Langguth B, Kreuzer PM, Kleinjung T, De Ridder D. Tinnitus: causes and clinical management. Lancet Neurol. 2013;12(9):920-30. https://doi.org/10.1 016/S1474-4422(13)70160-1. 
4. Henry JA, Reavis KM, Griest SE, Thielman EJ, Theodoroff SM, Grush LD, et al. Tinnitus: an epidemiologic perspective. Otolaryngol Clin North Am. 2020; 53(4):481-99. https://doi.org/10.1016/j.otc.2020.03.002.

5. Baguley D, McFerran D, Hall D. Tinnitus. Lancet. 2013;382(9904):1600-7. https://doi.org/10.1016/S0140-6736(13)60142-7.

6. Langguth $B$, Elgoyhen $A B$, Cederroth $C R$. Therapeutic approaches to the treatment of tinnitus. Annu Rev Pharmacol Toxicol. 2019;59(1):291-313. https://doi.org/10.1146/annurev-pharmtox-010818-021556.

7. Bhatt JM, Bhattacharyya N, Lin HW. Relationships between tinnitus and the prevalence of anxiety and depression. The Laryngoscope. 2017;127(2):466-9. https://doi.org/10.1002/lary.26107.

8. Fioretti $A B$, Fusetti M, Eibenstein A. Association between sleep disorders, hyperacusis and tinnitus: evaluation with tinnitus questionnaires. Noise Health. 2013;15(63):91-5. https://doi.org/10.4103/1463-1741.110287.

9. Pattyn T, Van Den Eede F, Vanneste S, Cassiers L, Veltman DJ, Van De Heyning P, et al. Tinnitus and anxiety disorders: a review. Hear Res. 2016; 333:255-65. https://doi.org/10.1016/j.heares.2015.08.014.

10. Salazar JW, Meisel K, Smith ER, Quiggle A, McCoy DB, Amans MR. Depression in patients with tinnitus: a systematic review. Otolaryngol Head Neck Surg. 2019;161(1):28-35. https://doi.org/10.1177/0194599819835178.

11. Langguth B. Non-invasive neuromodulation for tinnitus. J Audiol Otol. 2020; 24(3):113-8. https://doi.org/10.7874/jao.2020.00052.

12. Peter $\mathrm{N}$, Liyanage $\mathrm{N}$, Pfiffner $\mathrm{F}$, Huber $\mathrm{A}$, Kleinjung $\mathrm{T}$. The influence of cochlear implantation on tinnitus in patients with single-sided deafness: a systematic review. Otolaryngol Neck Surg. 2019;161(4):576-88. https://doi. org/10.1177/0194599819846084.

13. Searchfield GD, Durai M, Linford T. A state-of-the-art review: personalization of tinnitus sound therapy. Front Psychol. 2017;8:1599. https://doi.org/10.33 89/fpsyg.2017.01599.

14. Wang H, Tang D, Wu Y, Zhou L, Sun S. The state of the art of sound therapy for subjective tinnitus in adults. Ther Adv Chronic Dis. 2020;11: 2040622320956426. https://doi.org/10.1177/2040622320956426.

15. Hoare DJ, Edmondson-Jones M, Sereda M, Akeroyd MA, Hall D. Amplification with hearing aids for patients with tinnitus and co-existing hearing loss. Cochrane Database Syst Rev 2014(1):CD010151. https://doi. org/10.1002/14651858.CD010151.pub2.

16. Sereda M, Xia J, El Refaie A, Hall DA, Hoare DJ. Sound therapy (using amplification devices and/or sound generators) for tinnitus. Cochrane Database Syst Rev. 2018;12(12):CD013094. https://doi.org/10.1002/14651858. CD013094.pub2.

17. Cima RFF, Mazurek B, Haider H, Kikidis D, Lapira A, Noreña A, et al. A multidisciplinary European guideline for tinnitus: diagnostics, assessment, and treatment. HNO. 2019;67(S1):10-42. https://doi.org/10.1007/s00106-0190633-7.

18. Cima RFF, Maes IH, Joore MA, Scheyen DJWM, El Refaie A, Baguley DM, et al. Specialised treatment based on cognitive behaviour therapy versus usual care for tinnitus: a randomised controlled trial. Lancet Lond Engl. 2012;379(9830):1951-9. https://doi.org/10.1016/S0140-6736(12)60469-3.

19. Fuller T, Cima R, Langguth B, Mazurek B, Vlaeyen JW, Hoare DJ. Cognitive behavioural therapy for tinnitus. Cochrane Database Syst Rev. 2020;1(1): CD012614. https://doi.org/10.1002/14651858.CD012614.pub2.

20. Kikidis D, Vassou E, Schlee W, lliadou E, Markatos N, Triantafyllou A, et al. Methodological aspects of randomized controlled trials for tinnitus: a systematic review and how a decision support system could overcome barriers. J Clin Med. 2021;10(8):1737. https://doi.org/10.3390/jcm10081737.

21. Simoes JP, Daoud E, Shabbir M, Amanat S, Assouly K, Biswas R, et al. Multidisciplinary tinnitus research: challenges and future directions from the perspective of early stage researchers. Front Aging Neurosci. 2021;13: 647285. https://doi.org/10.3389/fnagi.2021.647285.

22. Zenner H-P, Delb W, Kröner-Herwig B, Jäger B, Peroz I, Hesse G, et al. A multidisciplinary systematic review of the treatment for chronic idiopathic tinnitus. Eur Arch Otorhinolaryngol. 2017;274(5):2079-91. https://doi.org/10.1 007/s00405-016-4401-y.

23. Hesse G. Evidence and evidence gaps in tinnitus therapy. GMS Curr Top Otorhinolaryngol Head Neck Surg. 2016;15:Doc04. https://doi.org/10.3205/ cto000131.

24. Cederroth CR, Gallus S, Hall DA, Kleinjung T, Langguth B, Maruotti A, et al. Editorial: towards an understanding of tinnitus heterogeneity. Front Aging Neurosci. 2019;11:53. https://doi.org/10.3389/fnagi.2019.00053.

25. Van de Heyning P, Gilles A, Rabau S, Van Rompaey V. Subjective tinnitus assessment and treatment in clinical practice: the necessity of personalized medicine. Curr Opin Otolaryngol Head Neck Surg. 2015;23(5):369-75. https://doi.org/10.1097/MOO.0000000000000183.

26. Simoes J, Neff P, Schoisswohl S, Bulla J, Schecklmann M, Harrison S, et al. Toward personalized tinnitus treatment: an exploratory study based on internet crowdsensing. Front Public Health. 2019;7:157. https://doi.org/10.33 89/fpubh.2019.00157.

27. Schaaf $H$, Eichenberg $C$, Kastellis $G$, Hesse G. Treatment of tinnitus needs a combined neurootological and psychosomatic approach. Otolaryngol Pol. 2010;64(2):78-82. https://doi.org/10.1016/S0030-6657(10)70039-1.

28. Henry JA, McMillan G, Dann S, Bennett K, Griest S, Theodoroff S, et al. Tinnitus management: randomized controlled trial comparing extendedwear hearing aids, conventional hearing aids, and combination instruments. J Am Acad Audiol. 2017;28(06):546-61. https://doi.org/10.3766/jaaa.16067.

29. Henry JA, Frederick M, Sell S, Griest S, Abrams H. Validation of a novel combination hearing aid and tinnitus therapy device. Ear Hear. 2015;36(1): 42-52. https://doi.org/10.1097/AUD.0000000000000093.

30. Tutaj L, Hoare DJ, Sereda M. Combined amplification and sound generation for tinnitus: a scoping review. Ear Hear. 2018;39(3):412-22. https://doi.org/1 0.1097/AUD.0000000000000516.

31. Conlon B, Langguth B, Hamilton C, Hughes S, Meade E, Connor CO, et al. Bimodal neuromodulation combining sound and tongue stimulation reduces tinnitus symptoms in a large randomized clinical study. Sci Transl Med. 2020;12(564):eabb2830. https://doi.org/10.1126/scitranslmed.abb2830.

32. Marks KL, Martel DT, Wu C, Basura GJ, Roberts LE, Schvartz-Leyzac KC, et al. Auditory-somatosensory bimodal stimulation desynchronizes brain circuitry to reduce tinnitus in guinea pigs and humans. Sci Transl Med. 2018;10(422): eaal3175. https://doi.org/10.1126/scitranslmed.aal3175.

33. Bauer CA, Berry JL, Brozoski TJ. The effect of tinnitus retraining therapy on chronic tinnitus: a controlled trial: a controlled trial of tinnitus retraining therapy. Laryngoscope Investig Otolaryngol. 2017;2(4):166-77. https://doi. org/10.1002/lio2.76.

34. Jastreboff PJ. 25 Years of tinnitus retraining therapy. HNO. 2015;63(4):307-11. https://doi.org/10.1007/s00106-014-2979-1.

35. Kreuzer PM, Poeppl TB, Bulla J, Schlee W, Lehner A, Langguth B, et al. A proof-of-concept study on the combination of repetitive transcranial magnetic stimulation and relaxation techniques in chronic tinnitus. J Neural Transm Vienna Austria 1996. 2016;123:1147-57. https://doi.org/10.1007/ s00702-016-1588-4.

36. Brüggemann P, Otto J, Lorenz N, Schorsch S, Szczepek AJ, Böcking B, et al. Long-term changes in multimodal intensive tinnitus therapy. HNO. 2018; 66(S1):34-8. https://doi.org/10.1007/s00106-017-0463-4.

37. Seydel C, Haupt H, Szczepek AJ, Klapp BF, Mazurek B. Long-term improvement in tinnitus after modified tinnitus retraining therapy enhanced by a variety of psychological approaches. Audiol Neurootol. 2010;15(2):6980. https://doi.org/10.1159/000231632.

38. Schlee W, Schoisswohl S, Staudinger S, Schiller A, Lehner A, Langguth B, et al. Towards a unification of treatments and interventions for tinnitus patients: the EU research and innovation action UNITI. Prog Brain Res. 2021; 260:441-51. https://doi.org/10.1016/bs.pbr.2020.12.005.

39. Schlee W, Langguth B, Pryss R, Allgaier J, Mulansky L, Vogel C, et al. Using big data to develop a clinical decision support system for tinnitus treatment. Berlin, Heidelberg: Springer; 2021. p. 1-15. https://doi.org/10.1 007/7854_2021_229

40. Chan A-W, Tetzlaff JM, Gøtzsche PC, Altman DG, Mann H, Berlin JA, et al. SPIRIT 2013 explanation and elaboration: guidance for protocols of clinical trials. BMJ. 2013;346(jan08 15):e7586. https://doi.org/10.1136/bmj.e7586.

41. Newman CW, Jacobson GP, Spitzer JB. Development of the tinnitus handicap inventory. Arch Otolaryngol Head Neck Surg. 1996;122(2):143-8. https://doi.org/10.1001/archotol.1996.01890140029007.

42. Meikle M, Henry J, Griest S, Stewart B, Abrams H, McArdle R, et al. The Tinnitus Functional Index: development of a new clinical measure for chronic, intrusive tinnitus. Ear Hear. 2011;33(2):153-76. https://doi.org/10.1 097/AUD.0b013e31822f67c0

43. Hiller W, Goebel G. Rapid assessment of tinnitus-related psychological distress using the Mini-TQ. Int J Audiol. 2004;43(10):600-4. https://doi.org/1 0.1080/14992020400050077.

44. Landgrebe M, Zeman F, Koller M, Eberl Y, Mohr M, Reiter J, et al. The Tinnitus Research Initiative (TRI) database: a new approach for delineation of tinnitus subtypes and generation of predictors for treatment outcome. BMC Med Inform Decis Mak. 2010;10(1):42. https://doi.org/10.1186/1472-6947-1 $0-42$. 
45. Adamchic I, Tass PA, Langguth B, Hauptmann C, Koller M, Schecklmann M, et al. Linking the Tinnitus Questionnaire and the subjective Clinical Global Impression: Which differences are clinically important? Health Qual Life Outcomes. 2012;10(1):79. https://doi.org/10.1186/1477-7525-10-79.

46. Kroenke K, Spitzer RL. The PHQ-9: a new depression diagnostic and severity measure. Psychiatr Ann. 2002;32(9):509-15. https://doi.org/10.3928/00485713-20020901-06

47. Kroenke K, Spitzer RL, Williams JBW. The PHQ-9. J Gen Intern Med. 2001; 16(9):606-13. https://doi.org/10.1046/j.1525-1497.2001.016009606.x.

48. Genitsaridi E, Partyka M, Gallus S, Lopez-Escamez JA, Schecklmann M, Mielczarek M, et al. Standardised profiling for tinnitus research: The European School for Interdisciplinary Tinnitus Research Screening Questionnaire (ESIT-SQ). Hear Res. 2019;377:353-9. https://doi.org/10.1016/j. heares.2019.02.017

49. Langguth B, Goodey R, Azevedo A, Bjorne A, Cacace A, Crocetti A, et al. Consensus for tinnitus patient assessment and treatment outcome measurement: Tinnitus Research Initiative meeting, Regensburg, July 2006. Prog Brain Res. 2007;166:525-36. https://doi.org/10.1016/S0079-6123 (07)66050-6

50. Bläsing L, Goebel G, Flötzinger U, Berthold A, Kröner-Herwig B. Hypersensitivity to sound in tinnitus patients: an analysis of a construct based on questionnaire and audiological data. Int J Audiol. 2010;49(7):51826. https://doi.org/10.3109/14992021003724996.

51. Danner D, Rammstedt B, Bluemke M, Treiber L, Berres S, Soto C, et al. Die deutsche Version des Big Five Inventory 2 (BFI-2). Zusammenstellung Sozialwissenschaftlicher Items Skalen ZIS; 2016. https://doi.org/10.6102/zis24 7.

52. Nasreddine ZS, Phillips NA, Bédirian V, Charbonneau S, Whitehead V, Collin I, et al. The Montreal Cognitive Assessment, MoCA: a brief screening tool for mild cognitive impairment. J Am Geriatr Soc. 2005;53(4):695-9. https://doi. org/10.1111/j.1532-5415.2005.53221.x.

53. Schlee W, Hølleland S, Bulla J, Simoes J, Neff P, Schoisswohl S, et al. The effect of environmental stressors on tinnitus: a prospective longitudinal study on the impact of the COVID-19 pandemic. J Clin Med. 2020;9(9):2756. https://doi.org/10.3390/jcm9092756.

54. Saunders GH, Cienkowski KM, Forsline A, Fausti S. Normative data for the attitudes towards Loss of Hearing Questionnaire. J Am Acad Audiol. 2005; 16(09):637-52. https://doi.org/10.3766/jaaa.16.9.2

55. Fuller TE, Cima RFF, Van den Bussche E, Vlaeyen JWS. The Fear of Tinnitus Questionnaire: toward a reliable and valid means of assessing fear in adults with tinnitus. Ear Hear. 2019;40(6):1467-77. https://doi.org/10.1097/AUD. 0000000000000728.

56. Melcher JR, Kiang NY. Generators of the brainstem auditory evoked potential in cat. III: Identified cell populations. Hear Res. 1996;93(1-2):52-71. https://doi.org/10.1016/0378-5955(95)00200-6

57. Burkard R, Don M. Introduction to auditory evoked potentials. Handb. Clin. Audiol., 2015.

58. Chalak S, Kale A, Deshpande VK, Biswas DA. Establishment of normative data for monaural recordings of auditory brainstem response and its application in screening patients with hearing loss: a cohort study. J Clin Diagn Res JCDR. 2013;7:2677-9. https://doi.org/10.7860/JCDR/2013/6768.373 0 .

59. Watson DR. The effects of cochlear hearing loss, age and sex on the auditory brainstem response. Audiol Off Organ Int Soc Audiol. 1996;35(5): 246-58. https://doi.org/10.3109/00206099609071945.

60. Roberts LE. Residual inhibition. Prog Brain Res. 2007;166:487-95. https://doi. org/10.1016/S0079-6123(07)66047-6

61. Szczepek AJ, Frejo L, Vona B, Trpchevska N, Cederroth CR, Caria H, et al. Recommendations on collecting and storing samples for genetic studies in hearing and tinnitus research. Ear Hear. 2019;40(2):219-26. https://doi.org/1 0.1097/AUD.0000000000000614

62. Amanat S, Gallego-Martinez A, Sollini J, Perez-Carpena P, Espinosa-Sanchez $J M$, Aran I, et al. Burden of rare variants in synaptic genes in patients with severe tinnitus: an exome based extreme phenotype study. EBioMedicine. 2021;66:103309. https://doi.org/10.1016/j.ebiom.2021.103309.

63. Neff P, Zielonka L, Meyer M, Langguth B, Schecklmann M, Schlee W. Comparison of amplitude modulated sounds and pure tones at the tinnitus frequency: residual tinnitus suppression and stimulus evaluation. Trends Hear. 2019;23:2331216519833841. https://doi.org/10.1177/2331216519833 841
64. Schoisswohl S, Arnds J, Schecklmann M, Langguth B, Schlee W, Neff P. Amplitude modulated noise for tinnitus suppression in tonal and noise-like tinnitus. Audiol Neurotol. 2019;24(6):309-21. https://doi.org/10.1159/000504 593.

65. Stein A, Wunderlich R, Lau P, Engell A, Wollbrink A, Shaykevich A, et al. Clinical trial on tonal tinnitus with tailor-made notched music training. BMC Neurol. 2016;16(1):38. https://doi.org/10.1186/s12883-016-0558-7.

66. Keidser $\mathrm{G}$, Dillon H, Flax M, Ching T, Brewer S. The NAL-NL2 prescription procedure. Audiol Res. 2011;1(1):88-90. https://doi.org/10.4081/audiores.2 011.e24.

67. Cima RFF. Bothersome tinnitus: cognitive behavioral perspectives. HNO. 2018;66(5):369-74. https://doi.org/10.1007/s00106-018-0502-9.

68. Cima RFF, van Breukelen G, Vlaeyen JWS. Tinnitus-related fear: mediating the effects of a cognitive behavioural specialised tinnitus treatment. Hear Res. 2018;358:86-97. https://doi.org/10.1016/j.heares.2017.10.003.

69. Savage J, Waddell A. Tinnitus. BMJ Clin Evid. 2014;2014:0506.

70. Rhee J, Lee D, Suh MW, Lee JH, Hong Y-C, Oh SH, et al. Prevalence, associated factors, and comorbidities of tinnitus in adolescents. PLOS ONE. 2020;15(7):e0236723. https://doi.org/10.1371/journal.pone.0236723.

71. Nondahl DM, Cruickshanks KJ, Dalton DS, Klein BEK, Klein R, Schubert CR, et al. The impact of tinnitus on quality of life in older adults. J Am Acad Audiol. 2007:18(03):257-66. https://doi.org/10.3766/jaaa.18.3.7.

72. Cima RFF, Crombez G, Vlaeyen JWS. Catastrophizing and fear of tinnitus predict quality of life in patients with chronic tinnitus. Ear Hear. 2011;32(5): 634-41. https://doi.org/10.1097/AUD.0b013e31821106dd.

73. Haider HF, Bojić T, Ribeiro SF, Paço J, Hall DA, Szczepek AJ. Pathophysiology of subjective tinnitus: triggers and maintenance. Front Neurosci. 2018;12: 866. https://doi.org/10.3389/fnins.2018.00866.

74. Eggermont JJ. Pathophysiology of tinnitus. Prog Brain Res. 2007;166:19-35. https://doi.org/10.1016/S0079-6123(07)66002-6.

75. Kleinjung T, Langguth B. Avenue for future tinnitus treatments. Otolaryngol Clin North Am. 2020;53(4):667-83. https://doi.org/10.1016/j.otc.2020.03.013.

76. Mehdi M, Riha C, Neff P, Dode A, Pryss R, Schlee W, et al. Smartphone apps in the context of tinnitus: systematic review. Sensors. 2020;20(6):1725. https://doi.org/10.3390/s20061725.

77. Mehdi M, Stach M, Riha C, Neff P, Dode A, Pryss R, et al. Smartphone and mobile health apps for tinnitus: systematic identification, analysis, and assessment. JMIR MHealth UHealth. 2020;8(8):e21767. https://doi.org/10.21 96/21767.

78. Mehdi M, Dode A, Pryss R, Schlee W, Reichert M, Hauck FJ. Contemporary review of smartphone apps for tinnitus management and treatment. Brain Sci. 2020;10(11):867. https://doi.org/10.3390/brainsci10110867.

\section{Publisher's Note}

Springer Nature remains neutral with regard to jurisdictional claims in published maps and institutional affiliations.

\section{Ready to submit your research? Choose BMC and benefit from:}

- fast, convenient online submission

- thorough peer review by experienced researchers in your field

- rapid publication on acceptance

- support for research data, including large and complex data types

- gold Open Access which fosters wider collaboration and increased citations

- maximum visibility for your research: over $100 \mathrm{M}$ website views per year

At $\mathrm{BMC}$, research is always in progress.

Learn more biomedcentral.com/submissions 